\title{
Integrated microRNA, gene expression and transcription factors signature in papillary thyroid cancer with lymph node metastasis
}

Nurul-Syakima Ab Mutalib, Sri Noraima Othman, Azliana Mohamad Yusof, Shahrun Niza Abdullah Suhaimi, Rohaizak Muhammad, Rahman Jamal

Background: Papillary thyroid carcinoma (PTC) is the commonest thyroid malignancy originating from the follicle cells in the thyroid. Despite a good overall prognosis, certain high-risk cases as in those with lymph node metastasis (LNM) have progressive disease and poorer prognosis. MicroRNAs are a class of non-protein-coding, 19-24 nucleotides single-stranded RNAs which regulate gene expression and these molecules have been shown to play a role in LNM. The integrated analysis of miRNAs and gene expression profiles together with transcription factors (TFs) has been shown to improve the identification of functional miRNA-target gene-TF relationships, providing a more complete view of molecular events underlying metastasis process. Objectives: We reanalyzed The Cancer Genome Atlas (TCGA) datasets on PTC to identify differentially expressed miRNAs/genes in PTC patients with LNM-positive (LNM-P) versus lymph node negative (LNN) PTC patients and to investigate the miRNA-gene-TF regulatory circuit that regulate LNM in PTC. Results: PTC patients with LNM (PTC LNM-P) has significantly shorter diseasefree survival rate compared to PTC patients without LNM (PTC LNN) (Log-rank Mantel Cox test, $p=0.0049$ ). We identified 181 significantly differentially expressed miRNAs in PTC LNM-P versus PTC LNN; 110 were upregulated and 71 were downregulated. The five topmost deregulated miRNAs were hsa-miR-146b, hsa-miR-375, hsa-miR-31, hsa-miR-7-2 and hsa-miR-204. In addition, 395 miRNAs were differentially expressed between PTC LNM$P$ and normal thyroid while 400 miRNAs were differentially expressed between PTC LNN and normal thyroid. We found four significant enrichment pathways potentially involved in metastasis to the lymph nodes namely oxidative phosphorylation (OxPhos), cell adhesion molecules (CAMs), leukocyte transendothelial migration and cytokine-cytokine receptor interaction. OxPhos was the most significantly perturbed pathway ( $p=4.70 E-06$ ) involving downregulation of 90 OxPhos-related genes. Significant interaction of hsa-miR-301b with HLF, HIF and REL/NFkB transcription factors were identified exclusively in PTC LNM-P versus PTC LNN. Conclusion: We found evidence of five miRNAs differentially expressed in PTC LNM-P. Alteration in OxPhos pathway could be the central event in metastasis to the 
lymph node in PTC. We postulate that hsa-miR-301b might be involved in regulating LNM in PTC via interactions with HLF, HIF and REL/NFkB. To the best of our knowledge, the roles of these TFs have been studied in PTC but the precise role of this miRNA with these TFs in LNM in PTC has not been investigated. 
1 Integrated microRNA, gene expression and transcription factors signature in papillary

2

\title{
thyroid cancer with lymph node metastasis
}

\author{
Nurul-Syakima Ab Mutalib ${ }^{1 * \#}$, Sri Noraima Othman ${ }^{1 \#}$, Azliana Mohd Yusof ${ }^{1}$, Shahrun Niza Abdullah \\ Suhaimi², Rohaizak Muhammad $^{2}$, Rahman Jamal ${ }^{1}$
}

${ }^{1}$ UKM Medical Molecular Biology Institute (UMBI), Universiti Kebangsaan Malaysia, Jalan Yaacob Latiff, 56000 Cheras, Kuala Lumpur, Malaysia

${ }^{2}$ Department of Surgery, Faculty of Medicine, Universiti Kebangsaan Malaysia, Jalan Yaacob Latiff, 56000 Cheras, Kuala Lumpur, Malaysia

\section{Introduction}

Papillary thyroid carcinoma (PTC) is the most common malignancy originating from the thyroid. Although the prognosis of PTC is generally good with a high 5-year survival rate, cases demonstrating certain clinicopathological parameters are progressive, have poorer prognosis and are considered as highrisk (Ito et al., 2009). Numerous classification systems for thyroid carcinoma have been established in order to classify high-risk cases such as AMES (Cady and Rosai, 1988), AGES (Hay et al., 1987), MACIS (Hay et al., 1993) as well as TNM (Sobin and Wittekind, 2002; AJCC 2010). The TNM classification is the most recent classification system and is based on size and extrathyroid extension (T), lymph node involvement $(\mathrm{N})$, distant metastasis $(\mathrm{M})$ and patient's age.

MicroRNAs (miRNAs), firstly identified in Caenorhabditis elegans, are a class of endogenous (nonprotein-coding), 19-24 nucleotides single-stranded RNAs that derive from a stem-loop precursor to inhibit gene expression by binding primarily to the 3'-UTR of specific 'target' messenger RNA (mRNAs). MiRNAs that bind with perfect or nearly perfect complementarity to protein-coding mRNA sequences 
24 induce the RNA-mediated interference (RNAi) pathway, resulting in the disruption of mRNA stability 25 and/or translation (Bartel 2009). Dysregulation of miRNAs expression in human cancers have been 26 demonstrated by many studies (Iorio and Croce, 2012). Through expression profiling studies, miRNAs

27 were shown to be linked to tumor development, tumor progression, and response to treatment, signifying 28 their potential use as biomarkers for diagnosis and prognosis (Iorio and Croce, 2012). MiRNAs have also 29 been shown function as biomarkers in predicting lymph node metastasis (LNM). There was a positive 30 correlation between high hsa-miR-21 expression with tumor stage and LNM in patients with breast cancer 31 (Yan et al., 2008), and the development of distant metastases in colorectal cancer patients (Slaby et al., 32 2007). Most recently, hsa-miR-1207-5p was suggested as a useful biomarker in the prediction of LNM in 33 gastric cancer (Huang et al., 2015) and head and neck cancer (de Carvalho et al., 2015).

The current approach of miRNA target gene prediction via in silico analysis is built upon sequence similarity search and thermodynamic stability (Alexiou et al., 2009). Nevertheless, it is acknowledged that the results of in silico target prediction algorithms suffer from very low specificity (Alexiou et al., 2009). The combination of in silico target predictions with miRNA and gene expression profiles has been proven to improve the identification of functional miRNA-target gene relationships (Nunez-Iglesias et al., 2010; Ma et al., 2011). As miRNAs act prevalently through degradation of the target genes, expression profiles of miRNA and target genes/transcripts are predicted to be inversely correlated (Bisognin et al., 2012). Another regulatory component, the transcription factors (TF), has also been shown to activate or repress miRNA expression level, further adding to the complexity of gene regulation. Efforts have been made to comprehend the mechanism of miRNAs in decreasing target genes expression; however the study of miRNA regulation by TFs (TF-miRNA regulation) is rather limited (Wang et al., 2010a).

The Cancer Genome Atlas (TCGA) Research Network recently published a molecular characterization of 507 PTCs and 59 matched normal adjacent tissues with respect to genomic, transcriptomic and proteomic 
49 thus creating a comprehensive dataset of PTC samples. Through unsupervised clustering methods, TCGA

50 yielded six subtypes for miRNA expression and five for gene expression. However, miRNA and gene

51 expression profiles between PTC with and without LNM were not comprehensively discussed. Here we

52 reanalyzed these TCGA datasets on PTC with the aim of identifying differentially expressed miRNAs/genes

53 in PTC patients with LNM-positive (LNM-P) as compared to lymph node negative (LNN) PTC patients

54 and to investigate the miRNA-gene-TF regulatory circuit that governs LNM in PTC.

\section{Materials and Methods}

\section{TCGA papillary thyroid cancer dataset}

We used the TCGA-generated microRNA sequencing (miRNAseq) and mRNAseq data for 495 tumors and 59 normal thyroid samples (Cancer Genome Atlas Research Network 2014). Metadata containing clinical information including $B R A F$ V600E mutation status was obtained from cBioPortal (http://www.cbioportal.org/study.do? cancer_study id=thca tcga_pub\#clinical) while miRNAseq and mRNAseq of 507 PTC patients were obtained from the TCGA Data Portal (https://tcgadata.nci.nih.gov/tcga/dataAccessMatrix.htm) [accessed from March 27, 2015 to May 25, 2015]. Information were available for 507 PTC patients. The list of patients from the metadata was then filtered for PTC patients with N0, N1, N1a, and N1b, resulting in a total of 421 PTC patients out of the 507 patients (86 patients were excluded due to unavailability of node status). The clinical parameters are presented in 67 Table 1.

Only samples with paired miRNAseq and mRNAseq data were selected, resulting in exclusion of additional three patients. In the end, we obtained a total of 418 patients' dataset which includes 213 patients with PTC LNN (N0) and 205 PTC LNM-P (53 patients with N1, 86 patients with N1a, 66 patients with

N1b) (Supplementary Table 1). Combined with 59 normal thyroid tissues, the total of datasets included in 
72 this study were 477 . The miRNA and gene expression datasets consisting of 1046 human miRNAs and

7320531 genes, respectively, were used for subsequent analysis.

74

75

76

77

\section{Survival analyses}

Kaplan-Meier survival analysis was carried out on disease-free and overall survival duration of TCGA PTC patients for whom follow-up details were available. Overall survival is defined as the duration from the date of diagnosis to death (due to all causes) while disease-free survival is defined as the duration from the date of the diagnosis to the date of recurrence, second cancer, or death due to all causes (whichever occurred first) (Schvartz et al., 2012). Curves were compared by univariate (log-rank) analysis. Statistical analyses were performed using GraphPad Prism version 6 (GraphPad, San Diego, CA, USA). P values $\leq$ 0.05 were considered significant.

\section{Clinical specimen and total RNA isolation}

Ten fresh frozen tumour-adjacent normal PTC tissues specimens from UKMMC-UMBI Biobank were subjected to cryosectioning and Haematoxylin and Eosin (H\&E) staining. This part of research was approved by the Universiti Kebangsaan Malaysia Research Ethics Committee (UKMREC) (reference: UKM 1.5.3.5/244/UMBI-2015-002). A written informed consent had been signed by these 10 subjects included in validation phase according to institution's rules and regulations. All the slides were reviewed by the pathologist to assess the percentage of tumour cells and normal cells. Only tumour tissues which contain $>80 \%$ cancer cells and normal tissues with $<20 \%$ necrosis were subjected to nucleic acid extraction. Total RNA including miRNA was isolated from the frozen samples using AllPrep DNA/RNA/miRNA Isolation Kit (Qiagen, Hilden, Germany) according to the manufacturer's protocol. The total RNA quality and quantity were assessed via absorbance spectrophotometry on a Nanodrop 1000 instrument (Thermo Scientific, Wilmington, DE, USA) and Qubit ${ }^{\mathrm{TM}}$ fluorometer (Invitrogen, USA). Integrity of RNA was 
96

97

98

99

100

101

102

103

104

105

106

107

108

109

110

111

112

113

114

115

116

117

118

119

assessed using Eukaryote Total RNA Nano chip on Bioanalyzer 2100 (Agilent Technologies, Santa Clara, USA). Only total RNA with RNA Integrity Number (RIN) of at least 6 were used for subsequent steps. Eukaryote Small RNA chip (Agilent Technologies, Santa Clara, USA) was used for determination of concentration and percentage of small RNA.

\section{Library preparation and next generation sequencing}

miRNA libraries were prepared using Illumina Truseq Small RNA library preparation kit (Illumina, SanDiego, USA) following manufacturer's protocol. Briefly, $3^{\prime}$ and 5' adapters were sequentially ligated to the ends of small RNAs fractionated from $1 \mu \mathrm{g}$ of total RNA, and reverse transcribed to generate cDNA. The cDNA was amplified using a common primer complementary to the $3^{\prime}$ adapter, and a primer containing 1 of 48 index sequences. Samples were size-selected (145-160 bp fragments) on a $6 \%$ polyacrylamide gel, purified, quantified and pooled for multiplexed sequencing. The resulting pooled libraries were normalized to $2 \mathrm{nM}$ and were hybridized to oligonucleotide-coated single-read flow cells for cluster generation using HiSeq ${ }^{\circledR}$ Rapid SR Cluster Kit v2 on Hiseq 2500. Subsequently the clustered pooled microRNA libraries were sequenced on the HiSeq 2500 for 50 sequencing cycles using HiSeq ${ }^{\circledR}$ Rapid SBS Kit v2 (50 Cycle). Base calling was performed using CASAVA (v.1.8.2) (Illumina, San Diego, USA) and short-read sequences in FASTQ format were used for downstream analysis.

\section{Bioinformatics analyses}

The miRNASeq and RNASeq V2 level 3 data from TCGA were used exclusively. The normalised expression (reads per million or RPM) of all miRNAs was $\log _{2}$-transformed and used for fold change calculation. The RNAseq by Expectation-Maximization (RSEM) values (from files with the extension .rsem.genes.results) were used to quantify messenger RNA (mRNA) expression levels. The RSEM algorithm is a statistical model that estimates RNA expression levels from RNA sequencing counts (Li and 
120 Dewey, 2011). We then performed the Students' unpaired t-test with a Benjamini Hochberg false discovery 121 rate (FDR) multiple testing correction and $\log _{2}$ fold change calculation using Bioconductor version 3.1

122 (BiocInstaller 1.18.2) (Gentleman et al., 2011) in R version 3.2.0 (R Development Core Team, 2008)

123 (Supplementary File 1 and 2). Downregulated genes will have negative $\log _{2}$ values while upregulated genes

124 will have positive $\log _{2}$ values. Statistical significance is denoted as $p \leq 0.05$. Heatmaps were created using

125 GeneE from the Broad Institute (http://www.broadinstitute.org/cancer/software/GENE-E) while Venn

126 diagrams were created using Venn online tool (http://bioinformatics.psb.ugent.be/webtools/Venn). All

127 other figures were created or labelled using Adobe Photoshop.

Analysis of the miRNAseq data from our in house experiment were performed using BaseSpace

aligner, the reads associated to mature miRNAs were counted and differential expression between experimental conditions were analysed using DESeq2 (Cordero et al., 2012). The expression, $\log _{2}$ fold were then extracted from the overall results.

\section{Pathway enrichment analysis and integrated analysis of miRNA and gene expression}

The functions and pathways of the differentially expressed genes were annotated and analysed using the annotation tools from the Database for Annotation, Visualization and Integrated Discovery (DAVID) (Huang et al., 2009a; Huang et al., 2009b) according to the steps described in these publications. The identified genes were also jointly annotated against the Kyoto Encyclopedia of Genes and Genomes (KEGG) database (Kanehisa and Goto, 2000). The genes that were annotated in the KEGG database as

142 being involved in signaling pathways were subjected to further analysis. Pathways with Benjamini-adjusted $143 \mathrm{p}$ value $\leq 0.05$ were considered to be statistically significant. 
performed in MAGIA2, a web tool for the integrated analysis of target predictions, miRNA and gene expression data (Bisognin et al., 2012). MiRNA target predictions include transcription factor binding sites

147 (TFBS) within miRNA and gene promoters. In this analysis, matched expression data matrices of 148 significantly dysregulated miRNAs and genes (BH adjusted $\mathrm{p}$ value $\leq 0.05$ ) were uploaded for integrated analysis. EntrezGene IDs and DIANA-microT (Maragkakis et al., 2009) target prediction algorithms were selected. Anticorrelated expressions were investigated between miRNA and their putative target genes using Pearson correlation measure.

\section{Results}

The effect of lymph node status on survival duration of TCGA PTC patients patients with LNM has significantly shorter disease-free survival rate compared to PTC patients without LNM (Log-rank Mantel Cox test, $\mathrm{p}=0.0049$; Figure 1B).

\section{Differentially expressed miRNAs}

We identified 181 miRNAs which were significantly differentially expressed in PTC LNM-P versus PTC LNN (BH corrected $\mathrm{p}$ value $\leq 0.05$ ). Among the 181 miRNAs significantly expressed in PTC LNM-P versus PTC LNN, 110 were upregulated and 71 were downregulated (Supplementary Table 2). Figure 2 illustrates a heatmap representing the expression levels of 181 deregulated miRNAs in PTC LNMP versus PTC LNN. The list of top deregulated miRNAs includes hsa-miR-146b, hsa-miR-375, hsa-miR31, hsa-miR-7-2 and hsa-miR-204 ( $\log _{2}$ fold change $1.7,1.3,1,-1.1$ and -1.3 , respectively, Figure 3$)$. On the other hand, 395 miRNAs were differentially expressed between PTC LNM-P and normal thyroid while 
167 168 169 170

400 miRNAs were differentially expressed between PTC LNN and normal thyroid (Supplementary Figure 1 and 2, respectively). The list of miRNAs significantly deregulated in PTC LNM-P and PTC LNN compared to normal thyroid is included in Supplementary Table 3.

We then determine the expression of these top deregulated miRNAs in a small set of validation experiment consisted of five pairs of tumour-adjacent normal from each PTC LNM-P and PTC LNN cases (total of 20 samples comprised of five PTC LNM-P, five PTC LNN and 10 adjacent normal thyroid tissues from each patient). As illustrated in Table 2, hsa-miR-146b was significantly upregulated in PTC LNM-P versus adjacent normal thyroid ( $\log _{2}$ fold change 6.0) and in PTC LNN versus adjacent normal thyroid $\left(\log _{2}\right.$ fold change 4.7). Similar trends were observed for hsa-miR-375, hsa-miR-31 and hsa-miR-204 in PTC LNM-P versus adjacent normal thyroid ( $\log _{2}$ fold change 3.6, 3.1 and -3.4, respectively, Table 2). However, downregulation of hsa-miR-7-2 in PTC LNM-P versus adjacent normal thyroid did not reach statistical significance. On the other hand, expression of hsa-miR-375, hsa-miR-7-2 and hsa-miR-204 in PTC LNN versus adjacent normal thyroid were in concordance with our analysis using the TCGA data $\left(\log _{2}\right.$ fold change 3.6, -2.3 and -2.7, Table 2).

These findings did not deviate much from our analysis using the TCGA PTC data with the exception to hsa-miR-7-2 in PTC LNM-P versus adjacent normal thyroid and hsa-miR-31 in PTC LNN versus adjacent normal thyroid which failed to reach statistical significance (Supplementary Table 2). This could be explained due to the fact that our validation samples were tumour-adjacent normal tissues while TCGA PTC specimens were of unpaired normal tissues. However, the differential expression of these five top deregulated miRNAs did not reach statistical significant when we compared between PTC LNM-P and PTC LNN (Table 2). This might be due to small sample size in our validation study.

\section{Differentially expressed genes}


192 There were 4135 upregulated and 4476 downregulated genes in PTC LNM-P relative to PTC LNN. By

193 increasing the stringency of selection to genes with $\log _{2}$ fold change $\geq 1$ or $\leq-1,407$ genes were identified

194 as strongly deregulated. Among the strongly deregulated genes were SFTPB, CLDN10, DIO1 and MT1G

195 ( $\log _{2}$ fold change 3.1, 2.9, -2.2 and -2.5 respectively, Supplementary Table 4). Various cancer-related genes

196 were also differentially expressed significantly, including $B R A F, B R C A 2, V E G F A, V E G F B, R E T, P I K 3 C A$,

197 CTNNB1 and GNAS (Supplementary Table 4).

\section{Enriched pathways in PTC LNM-P}

The significantly dysregulated genes in PTC LNM-P versus PTC LNN were mainly enriched in 12

KEGG pathways including oxidative phosphorylation (OxPhos), Parkinson's disease, focal adhesion,

202

203

204

205

206

207

208

209

210

211

212

213

214

Alzheimer's disease, valine, leucine and isoleucine degradation, pathways in cancer, cell adhesion molecules (CAMs), leukocyte transendothelial migration, cytokine-cytokine receptor interaction, small cell lung cancer, Huntington's disease and extracellular matrix receptor interaction (Figure 4A). When we overlapped the results from the three comparison groups (PTC LNM-P versus PTC LNN, PTC LNM-P versus normal thyroid and PTC LNN versus normal thyroid), four unique pathways potentially involved in metastasis to the lymph nodes were significantly enriched, namely, oxidative phosphorylation (OxPhos), cell adhesion molecules (CAMs), leukocyte transendothelial migration and cytokine-cytokine receptor interaction pathways (Figure 4A). The oxidative phosphorylation pathway was the most significantly perturbed $(\mathrm{p}=4.70 \mathrm{E}-06)$ with general downregulation of 90 OxPhos-related genes (Figure 5). Focal adhesion and pathways in cancer were commonly enriched in all the three group comparisons. Pathways in cancer is a collection of general cancer-related pathways and is an indication that many essential carcinogenic processes may be under the influence of dysregulated miRNAs (Pizzini et al., 2013). On the other hand, ECM-receptor interaction pathway and the valine, leucine and isoleucine degradation pathway 
215

216

217

218

219

220

221

222

223

224

225

226

227

228

229

230

231

232

233

234

235

236

237

238

were commonly enriched only in PTC LNM-P versus PTC LNN and PTC LNM-P versus normal thyroid but were not enriched in PTC LNN versus normal thyroid (Figure 4B).

\section{Integrated mixed regulatory circuits, involving miRNAs, genes and TFs in PTC LNM-P}

To obtain a more comprehensive insight into the molecular circuits behind LNM in PTC, we focused on functional miRNA-target relationships by performing an in silico integration between differentially expressed miRNAs and genes using MAGIA2. Transcription factors-miRNA (TF-miRNA) prediction was based on mirGen2.0 database (Friard et al., 2010) and TransmiR (Wang et al., 2010a), whereas the TF-gene interactions were acquired from the 'TFBS conserved' track of the University of California Santa Cruz (UCSC) genome annotation for humans (version hg19) (Bisognin et al., 2012). Our results show that 12 miRNAs are involved in the strongest 200 interactions and they were identified as significant by MAGIA2. Hsa-miR-147b, hsa-miR-301b, hsa-miR-375, hsa-miR-496, hsa-miR-543, hsamiR-577, hsa-miR-765, hsa-miR-892a, hsa-miR-934, hsa-miR-935, hsa-miR-940 and hsa-miR-944 were predicted to activate or inhibit 3746 genes and 1987 TFs (Figure 6). Hsa-miR-577 and hsa-miR-147b consistently appeared in the top 20 regulatory circuits across all group comparisons. Interestingly, hsa-miR301b appeared in both of the top 20 circuits in PTC LNM-P versus PTC LNN or normal thyroid but was absent in PTC LNN versus normal thyroid (Supplementary figure 3).

\section{Discussion}

In this study, we explored the landscape of miRNA and mRNA expression of PTC using data obtained from the TCGA THCA project aiming to identify key pathways involved in lymph node metastasis. Our analysis revealed 110 upregulated miRNAs and 71 downregulated miRNAs in PTC LNM-P versus PTC LNN. The top deregulated miRNAs includes hsa-miR-146b, hsa-miR-375, hsa-miR-31, hsamiR-7-2 and hsa-miR-204. Our findings are supported by several other similar studies, and in particular 
239 hsa-miR-146b, which was reported to be upregulated in PTC LNM-P versus PTC LNN (Lee et al., 2013;

240 Yang et al., 2013; Acibucu et al., 2014; Deng et al., 2015).

241 Hsa-miR-146 is one of the widely studied miRNAs in thyroid cancers and has been shown to be 242 frequently upregulated in PTC (He et al., 2005; Pallante et al., 2006; Tetzlaff et al., 2007; Chen et al., 243 2008; Yip et al., 2011; Chou et al., 2010; Chou et al., 2013; Sun et al., 2013), anaplastic thyroid cancer 244 (Fassina et al., 2014) and follicular thyroid cancer (FTC) (Wojtas et al., 2014). Functional analyses of hsamiR-146 revealed its involvement in various cellular functions including migration, invasion, proliferation, colony-forming ability, cell cycle, and resistance to chemotherapy-induced apoptosis in $B R A F$-mutated cell

247 lines (Chou et al., 2013; Deng et al., 2015; Geraldo et al., 2012). Using multivariate logistic regression analysis, Chou and colleagues demonstrated that increased hsa-miR-146b expression is one of the independent risk factors for poor prognosis in PTC, implicating the potential of this miRNA as a prognostic marker (Chou et al., 2013). which has been reported as the direct targets of this miRNA in PTC. Geraldo et al. (2012) reported SMAD4, an important member of the transforming growth factor $\beta$ (TGF- $\beta$ ) signaling pathway, as the target of hsamiR-146b-5p (Geraldo et al., 2012). The direct binding of hsa-miR-146b-5p on the SMAD4 UTR was confirmed via a luciferase reporter assay and the inhibition of hsa-miR-146b-5p expression resulted in significantly increased SMAD4 gene and protein expression levels in the human PTC cell lines. Furthermore, the inhibition of hsa-miR-146b-5p increased the cellular response to the TGF- $\beta$ antiproliferative signal, leading to significant reduction of cell proliferation (Geraldo et al., 2012). In a more recent study, the Zinc Ring Finger 3 (ZNRF3) gene was revealed as a direct target of hsa-miR-146b-5p and this miRNA was shown to stimulate cell migration, invasion and epithelial-to-mesenchymal transition (EMT) by downregulating ZNRF3 (Deng et al., 2015). Another study showed that ZNRF3 inhibits Wnt 
264 via suppression of $Z N R F 3$, causing enhanced Wnt/ $\beta$-catenin signaling. These findings revealed a novel 265 mechanism of hsa-miR-146b-5p in mediating the induction of EMT and implied the role of ZNRF3 as a 266 tumor suppressor in PTC (Deng et al., 2015). Additional efforts to identify genes controlled by hsa-miR-

$267146 \mathrm{~b}$ associated with LNM will eventually revealed new biomarkers that can be utilized to correlate with 268 disease outcome in PTC patients. Hsa-miR-204 expression in PTC LNM-P is significantly lower than in PTC LNN. This is the first report showing the downregulation of hsa-miR-204 in PTC LNM-P. This miRNA was also downregulated in PTC compared to adjacent normal thyroid tissue and noncancerous thyroid (Swierniak et al., 2013). It is likely that hsa-miR-204 is downregulated in PTC compared to normal or benign thyroid disease and is further supressed when lymph node metastasis occurs. This miRNA is known as a tumor suppressor miRNA and is downregulated in various cancers including renal clear cell carcinoma (Gowrishankar et al., 2014), minimal deviation adenocarcinoma (MDA) of uterine cervix (Lee et al., 2014) and breast cancer (Li et al., 2014). This miRNA has also been shown to have a prognostic value; low level of hsa-miR-204-5p expression was correlated with LNM, advanced stage and low survival rate in endometrial cancer (Bao et al., 2013), and also poor prognosis in colorectal cancer (Yin et al., 2014). In vitro functional analyses revealed the involvement of hsa-miR-204 in inhibiting the clonogenic growth, migration and invasion of endometrial carcinoma cells (Bao et al., 2013). In addition, restoration of hsa-miR-204-5p expression supressed cell proliferation, migration, invasion and induced apoptosis and chemotherapeutic sensitivity in colorectal cancer cell (Yin et al., 2014). one study investigating the functional role of hsa-miR-204 in PTC (Liu et al., 2015). Enforced expression of hsa-miR-204-5p inhibited cell proliferation and induced apoptosis and cell cycle arrest in PTC cell lines (TCP-1 and BCPAP). In addition, hsa-miR-204-5p also inhibits PTC cell tumorigenicity in vivo (Liu et al., 2015). Bioinformatics prediction analyses using three algorithms (miRanda, Pictar, and TargetScan) revealed the insulin-like growth factor-binding protein 5 (IGFBP5), a gene playing an essential role in 
289

290

291

292

293

294

295

296

297

298

299

300

301

302

303

304

305

306

307

308

309

310

311

312

313

carcinogenesis (Beattie et al., 2006), as a potential target of hsa-miR-204-5p. Luciferase reporter assay confirmed the direct binding of hsa-miR-204-5p to the 3' UTR of IGFBP5 (Liu et al., 2015). In the same study, hsa-miR-204-5p and IGFBP5 expression were also shown to be inversely correlated. Their findings confirmed the role of hsa-miR-204-5p as a tumor suppressor in PTC and revealed the potential use of this miRNA as a therapeutic agent in the treatment of PTC.

In an attempt to identify genes and pathways associated with mortality in PTC, Nilubol et al. (2011) performed genome-wide expression (GWE) analysis in 64 PTC patients and identified the oxidative phosphorylation pathway as one of the significantly perturbed pathways. In addition, Lee and colleagues also showed that the expression of OxPhos gene sets was significantly lower in primary PTC than in matched normal thyroid tissue (Lee et al., 2015a). Our findings revealed a similar trend with OxPhos genes being significantly downregulated in PTCs versus normal thyroid tissues as well as in PTC LNM-P versus PTC LNN. However, significant enrichment of OxPhos pathway was only observed in PTC LNM-P compared to PTC LNN. Alteration in metabolic processes has been considered as an indispensable component of malignant transformation (Lee et al., 2015a) thus the involvement of oxidative phosphorylation in LNM in PTC necessitates further investigation.

Oxidative phosphorylation is a process whereby an adenosine triphosphate (ATP) is produced as a result of electrons transfer from nicotinamide adenine dinucleotide (NADH) or flavin-adenine dinucleotide $\left(\mathrm{FADH}_{2}\right)$ to oxygen by a series of electron carriers (Berg et al., 2002). The thyroid gland is an endocrine organ with a high energy consumption and oxidative processes are crucial for thyroid hormone synthesis (Lee et al., 2015b). The mitochondria is responsible for providing $90 \%$ of the cellular energy necessary for various biological functions through oxidative phosphorylation and plays an important role in energy metabolism in the normal thyroid gland and in thyroid tumors (Kim et al., 2012). The mitochondria is involved in many cell signaling pathways by playing crucial roles in apoptosis, cell proliferation and cellular $\mathrm{Ca}^{2+}$ homeostasis (Rustin 2002). Mitochondrial DNA (mtDNA) content was shown to be higher in PTC compared to the paired normal DNA and in normal controls (Mambo et al., 2005). Despite 
314 advancement in the elucidation of molecular events underlying thyroid carcinogenesis in the last decade,

315 the function and nature of energy metabolism in thyroid cancer remain unclear (Lee et al., 2015b).

316 In addition to oxidative phosphorylation, we also identified significant enrichment of other cancer-

317 related pathways such as cell adhesion molecules (CAMs), leukocyte transendothelial migration and

318 cytokine-cytokine receptor interaction pathways which were unique to PTC LNM-P versus PTC LNN.

319 Interestingly, these pathways were not significantly enriched when PTCs (LNM-P and LNN) were 320 compared to normal thyroid tissues. Taken together, it could be hypothesized that metastasis to the lymph

321 node in PTC occurred via changes in the aforementioned pathways. However, some pathways in our

322 analysis, such as valine, leucine and isoleucine degradation, could not be associated with oncogenesis or 323 metastasis and may need further investigation.

Our integrated analysis revealed hsa-miR-301b's presence in the top 20 circuits in both PTC LNM-

P versus PTC LNN and PTC LNM-P versus normal thyroid but was absent in PTC LNN versus normal thyroid despite significant downregulation with modest fold change $\left(\log _{2}\right.$ fold change of -0.3$)$. Hsa-miR301 is located in the intronic region of SKA2 (spindle and kinetochore associated complex subunit 2) and belongs to the hsa-miR-130 microRNA precursor family (Cao et al., 2010). In contrast to our findings, hsamiR-301 upregulation has been reported in various cancers of non-thyroid origins and given that a miRNA can act either as an oncomiR or tumor suppressor depending on the cellular context and tissue type (Garzon et al., 2009), this observation is not unexpected. There is no evidence of hsa-miR-301b dysregulation in PTC so far, but it was reported to be upregulated in follicular thyroid adenoma compared to normal thyroid tissue (Rossing et al., 2012). It is also upregulated in CRC without LNM in comparison to paracancerous control (Wang et al., 2010b). The inhibition of hsa-miR-301 decreased breast cancer cell proliferation, 

are important for the regulation of genes. Majority of oncogenes and tumor suppressor genes encode the

340 TFs (Ell and Yang 2013). Dysregulation of oncogenic or tumor suppressive TFs could influence multiple 341 steps of the metastasis cascade, leading to cancer progression (Ell and Yang 2013). The involvement of TFs

342 in PTC has been investigated since decades ago and several thyroid-specific TFs have been identified

343 (Guazzi et al., 1990; Fabbro et al., 1994). Most recently, the glioma-associated oncogene homolog 1 (GLI1)

344 has been identified as a TF marker for LNM in PTC and it increases tumor aggressiveness via the Hedgehog 345 signaling pathway (Lee et al., 2015c). The hepatic leukemia factor (HLF) is the only TF which appeared in 346 the top 20 circuits of PTCs with or without LNM versus normal thyroid from our integrated analysis. On 347 the other hand, REL was identified in the top 20 circuits only in PTC LNM-P in comparison to PTC LNN and will be discussed further in the following section.

The HLF is a transcription factor that facilitates thyroid hormone activation from the thyroid hormone receptor/retinoid X receptor heterodimer to hypoxia-inducible factor (HIF-1 $\alpha$ ) (Otto and Fandrey 2008). Triiodothyronine (T3) indirectly increases HIF-1 $\alpha$ mRNA by increasing the expression of HLF, subsequently initiating the transcription of HIF-1 $\alpha$ transcription factor (Burrows et al., 2011). HIF is another transcription factor which acts under hypoxia and thus is active in a number of diseases associated with low oxygen environment including cancer (Burrows et al., 2011). In fact, the HIF-1 $\alpha$ protein was differentially expressed in primary thyroid cancers associated with advanced stage; its expression was supressed in normal thyroid tissue and was highest in the most aggressive dedifferentiated anaplastic thyroid carcinomas (ATCs) (Hanada et al., 2004), supporting its role for thyroid tumor aggressiveness, progression as well as metastasis. In addition, we also identified a significant involvement of REL/NFkB in lymph node metastasis of PTC which is in concordance with previously published data (Du et al., 2006). 
363

364

365

366

367

368

369

370

371

372

373

374

375

376

377

378

379

380

381

382

383

384

385

386

387

388

389

390

391

392

393

394

395

396

397

398

399

400

401

402

processes may provide an alternative therapeutic target for tackling metastasis or recurrence. In addition,

via the integrated analysis we discovered that hsa-miR-301b might be involved in promoting LNM in PTC via activation of HLF, HIF and REL/NFkB. As far as we know, the roles of these TFs have been explored in PTC; however the exact roles of this miRNA with these TFs in LNM in PTC have not been studied. Hence, further investigation is necessary for future research in order to completely unravel the mechanism of LNM in PTC.

\section{References}

Acibucu F, Dökmetaş HS, Tutar Y, Elagoz S, Kilicli F. Correlations between the expression levels of microRNA146b, 221, 222 and p27Kip1 protein mRNA and the clinicopathologic parameters in papillary thyroid cancers. Exp Clin Endocrinol Diabetes. 2014 Mar;122(3):137-43. doi: 10.1055/s-00341367025 .

AJCC: Thyroid. In: Edge SB, Byrd DR, Compton CC, Fritz AG, Greene FL, Trotti A, eds.: AJCC Cancer Staging Manual. 7th ed. New York, NY: Springer, 2010, pp 87-96.

Alexiou P, Maragkakis M, Papadopoulos GL, Reczko M, Hatzigeorgiou AG. Lost in translation: an assessment and perspective for computational microRNA target identification. Bioinformatics. 2009 Dec 1;25(23):3049-55. doi: 10.1093/bioinformatics/btp565.Bao W, Wang HH, Tian FJ, He XY, Qiu MT, Wang JY, Zhang HJ, Wang LH1, Wan XP. A TrkB-STAT3-miR-204-5p regulatory circuitry controls proliferation and invasion of endometrial carcinoma cells. Mol Cancer. 2013 Dec 9;12:155. doi: 10.1186/1476-4598-12-155.

Bartel, DP. Micrornas: Target Recognition and Regulatory Functions. Cell. 2009 Jan 23;136(2):215-33. doi: 10.1016/j.cell.2009.01.002.

Beattie J, Allan GJ, Lochrie JD, Flint DJ. Insulin-like growth factor-binding protein-5 (IGFBP-5): a critical member of the IGF axis. Biochem J. 2006 Apr 1;395(1):1-19.

Berg JM, Tymoczko JL, Stryer L. Biochemistry. 5th edition. New York: WH Freeman; 2002.

Bisognin A, Sales G, Coppe A, Bortoluzzi S, Romualdi C. MAGIA²: from miRNA and genes expression data integrative analysis to microRNA-transcription factor mixed regulatory circuits (2012 update). Nucleic Acids Res. 2012 Jul;40(Web Server issue). doi: 10.1093/nar/gks460.

Burrows N, Babur M, Resch J, Williams KJ, Brabant G. Hypoxia-inducible factor in thyroid carcinoma. J Thyroid Res. 2011;2011:762905. doi: 10.4061/2011/762905.Cady B, Rosai R. An expanded view of risk group definition in differentiated thyroid carcinoma. Surgery. 1988 Dec;104(6):947-53.

Cancer Genome Atlas Research Network. Integrated genomic characterization of papillary thyroid carcinoma. Cell. 2014 Oct 23;159(3):676-90. doi: 10.1016/j.cell.2014.09.050.

Cao G, Huang B, Liu Z, Zhang J, Xu H, Xia W, Li J, Li S, Chen L, Ding H, Zhao Q, Fan M, Shen B, Shao N. Intronic miR-301 feedback regulates its host gene, ska2, in A549 cells by targeting MEOX2 to affect ERK/CREB pathways. Biochem Biophys Res Commun. 2010 Jun 11;396(4):978-82. doi: 10.1016/j.bbrc.2010.05.037.

Chen YT, Kitabayashi N, Zhou XK, Fahey TJ 3rd, Scognamiglio T. MicroRNA analysis as a potential diagnostic tool for papillary thyroid carcinoma. Mod Pathol. 2008 Sep;21(9):1139-46. doi: 10.1038/modpathol.2008.105. 
403

404

405

406

407

408

409

410

411

412

413

414

415

416

417

418

419

420

421

422

423

424

425

426

427

428

429

430

431

432

433

434

435

436

437

438

439

440

441

442

443

444

445

446

447

448

449

450

451

452

453

Chou CK, Chen RF, Chou FF, Chang HW, Chen YJ, Lee YF, Yang KD, Cheng JT, Huang CC, Liu RT. miR-146b is highly expressed in adult papillary thyroid carcinomas with high risk features including extrathyroidal invasion and the BRAF(V600E) mutation. Thyroid. 2010 May;20(5):489-94. doi: 10.1089/thy.2009.0027.

Chou CK, Yang KD, Chou FF, Huang CC, Lan YW, Lee YF, Kang HY, Liu RT. Prognostic implications of miR-146b expression and its functional role in papillary thyroid carcinoma. J Clin Endocrinol Metab. 2013 Feb;98(2):E196-205. doi: 10.1210/jc.2012-2666.

Cordero F, Beccuti M, Arigoni M, Donatelli S, Calogero RA. Optimizing a massive parallel sequencing workflow for quantitative miRNA expression analysis. PLoS One. 2012;7(2):e31630. doi: 10.1371/journal.pone.0031630.

de Carvalho AC, Scapulatempo-Neto C, Maia DC, Evangelista AF, Morini MA, Carvalho AL, Vettore AL. Accuracy of microRNAs as markers for the detection of neck lymph node metastases in patients with head and neck squamous cell carcinoma. BMC Med. 2015 May 9;13:108. doi: 10.1186/s12916-0150350-3.

Deng X, Wu B, Xiao K, Kang J, Xie J, Zhang X, Fan Y. MiR-146b-5p promotes metastasis and induces epithelial-mesenchymal transition in thyroid cancer by targeting ZNRF3. Cell Physiol Biochem. 2015;35(1):71-82. doi: 10.1159/000369676.

Du ZX, Zhang HY, Gao DX, Wang HQ, Li YJ, Liu GL. Significance of VEGF and NF- $\kappa B$ expression in thyroid carcinoma. Chin J Clin Oncol. 2006;3:166-171. doi: 10.1007/s11805-006-0112-2.

Ell B, Kang Y. Transcriptional control of cancer metastasis. Trends Cell Biol. 2013 Dec;23(12):603-11. doi: $10.1016 /$ j.tcb.2013.06.001.

Fabbro D, Di Loreto C, Beltrami CA, Belfiore A, Di Lauro R, Damante G. Expression of thyroid-specific transcription factors TTF-1 and PAX-8 in human thyroid neoplasms. Cancer Res. 1994 Sep 1;54(17):4744-9.

Fassina A, Cappellesso R, Simonato F, Siri M, Ventura L, Tosato F, Busund LT, Pelizzo MR, Fassan M. A 4-MicroRNA signature can discriminate primary lymphomas from anaplastic carcinomas in thyroid cytology smears. Cancer Cytopathol. 2014 Apr;122(4):274-81. doi: 10.1002/cncy.21383.

Friard O, Re A, Taverna D, De Bortoli M, Corá D. CircuitsDB: a database of mixed microRNA/transcription factor feed-forward regulatory circuits in human and mouse. BMC Bioinformatics. 2010 Aug 23;11:435. doi: 10.1186/1471-2105-11-435.

Garzon R, Calin GA, Croce CM. MicroRNAs in Cancer. Annu Rev Med. 2009;60:167-79. doi: 10.1146/annurev.med.59.053006.104707.

Gentleman RC, Carey VJ, Bates DM, Bolstad B, Dettling M, Dudoit S, Ellis B, Gautier L, Ge Y, Gentry J, Hornik K, Hothorn T, Huber W, Iacus S, Irizarry R, Leisch F, Li C, Maechler M, Rossini AJ, Sawitzki G, Smith C, Smyth G, Tierney L, Yang JY, Zhang J. Bioconductor: open software development for computational biology and bioinformatics. Genome Biol. 2004;5(10):R80.

Geraldo MV, Yamashita AS, Kimura ET. MicroRNA miR-146b-5p regulates signal transduction of TGF$\beta$ by repressing SMAD4 in thyroid cancer. Oncogene. 2012 Apr 12;31(15):1910-22. doi: 10.1038/onc.2011.381.

Gowrishankar B, Ibragimova I, Zhou Y, Slifker MJ, Devarajan K, Al-Saleem T, Uzzo RG, Cairns P. MicroRNA expression signatures of stage, grade, and progression in clear cell RCC. Cancer Biol Ther. 2014 Mar 1;15(3):329-41. doi: 10.4161/cbt.27314.

Guazzi S, Price M, De Felice M, Damante G, Mattei MG, Di Lauro R. Thyroid nuclear factor 1 (TTF-1) contains a homeodomain and displays a novel DNA binding specificity. EMBO J. 1990 Nov;9(11):3631-9.

Hanada M, Feng J, Hemmings BA. Structure, regulation and function of PKB/AKT--a major therapeutic target. Biochim Biophys Acta. 2004 Mar 11;1697(1-2):3-16.

Hao HX, Xie Y, Zhang Y, Charlat O, Oster E, Avello M, Lei H, Mickanin C, Liu D, Ruffner H, Mao X, Ma Q, Zamponi R, Bouwmeester T, Finan PM, Kirschner MW, Porter JA, Serluca FC, Cong F. ZNRF3 promotes Wnt receptor turnover in an R-spondin-sensitive manner. Nature. 2012 Apr 29;485(7397):195-200. doi: 10.1038/nature11019.

Peer] reviewing PDF | (2015:10:7164:2:1:NEW 11 May 2016) 
454

455

456

457

458

459

460

461

462

463

464

465

466

467

468

469

470

471

472

473

474

475

476

477

478

479

480

481

482

483

484

485

486

487

488

489

490

491

492

493

494

495

496

497

498

499

500

501

502

503

Hay ID, Bergstrahl EJ, Goellner JR, Ebersold JR, Grant CS. Predicting outcome in papillary thyroid carcinoma: development of a reliable prognostic scoring system in a cohort of 1779 patients surgically treated at one institution during 1940 through 1989. Surgery. 1993 Dec;114(6):1050-7; discussion 1057-8.

Hay ID, Grant CS, Taylor WF, MaConahey WM. Ipsilateral lobectomy versus bilateral lobar resection in papillary thyroid carcinoma: a retrospective analysis of survical outcome using a novel prognostic scoring system. Surgery. 1987 Dec;102(6):1088-95.

He H, Jazdzewski K, Li W, Liyanarachchi S, Nagy R, Volinia S, Calin GA, Liu CG, Franssila K, Suster S, Kloos RT, Croce CM, de la Chapelle A. The role of microRNA genes in papillary thyroid carcinoma. Proc Natl Acad Sci U S A. 2005 Dec 27;102(52):19075-80.

Huang DW, Sherman BT, Lempicki RA. Bioinformatics enrichment tools: paths toward the comprehensive functional analysis of large gene lists. Nucleic Acids Res. 2009a;37(1):1-13. doi: 10.1093/nar/gkn923.

Huang DW, Sherman BT, Lempicki RA. Systematic and integrative analysis of large gene lists using DAVID Bioinformatics Resources. Nature Protoc. 2009b;4(1):44-57. doi: 10.1038/nprot.2008.211.

Huang KH, Lan YT, Fang WL, Chen JH, Lo SS, Li AF, Chiou SH, Wu CW, Shyr YM. The Correlation between miRNA and Lymph Node Metastasis in Gastric Cancer. Biomed Res Int. 2015;2015:543163. doi: $10.1155 / 2015 / 543163$.

Iorio MV, Croce CM. Microrna Dysregulation in Cancer: Diagnostics, Monitoring and Therapeutics. A Comprehensive Review. EMBO Mol Med. 2012 Mar;4(3):143-59. doi: 10.1002/emmm.201100209.

Ito Y, Fukushima M, Tomoda C, Inoue H, Kihara M, Higashiyama T, Uruno T, Takamura Y, Miya A, Kobayashi K, Matsuzuka F, Miyauchi A. Prognosis of patients with papillary thyroid carcinoma having clinically apparent metastasis to the lateral compartment. Endocr J. 2009;56(6):759-66.

Kanehisa M, Goto S. KEGG: kyoto encyclopedia of genes and genomes. Nucleic Acids Res. 2000 Jan 1;28(1):27-30.

Kanehisa M, Sato Y, Kawashima M, Furumichi M, Tanabe M. KEGG as a reference resource for gene and protein annotation. Nucleic Acids Res. 2016 Jan 4;44(D1):D457-62. doi: 10.1093/nar/gkv1070.

Kim SJ, Kwon MC, Ryu MJ, Chung HK, Tadi S, Kim YK, Kim JM, Lee SH, Park JH, Kweon GR, Ryu SW, Jo YS, Lee CH, Hatakeyama H, Goto Y, Yim YH, Chung J, Kong YY, Shong M. CRIF1 is essential for the synthesis and insertion of oxidative phosphorylation polypeptides in the mammalian mitochondrial membrane. Cell Metab. 2012 Aug 8;16(2):274-83. doi: 10.1016/j.cmet.2012.06.012.

Lee H, Kim KR, Cho NH, Hong SR, Jeong H, Kwon SY, Park KH, An HJ, Kim TH, Kim I, Yoon HK, Suh KS, Min KO, Choi HJ, Park JY, Yoo CW, Lee YS, Lee HJ, Lee WS, Park CS, Lee Y; Gynecological Pathology Study Group of the Korean Society of Pathologists. MicroRNA expression profiling and Notch1 and Notch2 expression in minimal deviation adenocarcinoma of uterine cervix. World J Surg Oncol. 2014 Nov 8;12:334. doi: 10.1186/1477-7819-12-334.

Lee J, Seol MY, Jeong S, Lee CR, Ku CR, Kang SW, Jeong JJ, Shin DY, Nam KH, Lee EJ, Chung WY, Jo YS. A metabolic phenotype based on mitochondrial ribosomal protein expression as a predictor of lymph node metastasis in papillary thyroid carcinoma. Medicine (Baltimore). 2015a Jan;94(2):e380. doi: 10.1097/MD.0000000000000380.

Lee J, Chang JY, Kang YE, Yi S, Lee MH, Joung KH, Kim KS, Shong M. Mitochondrial Energy Metabolism and Thyroid Cancers. Endocrinol Metab (Seoul). 2015b Jun;30(2):117-23. doi: 10.3803/EnM.2015.30.2.117.

Lee J, Jeong S, Lee CR, Ku CR, Kang SW, Jeong JJ, Nam KH, Shin DY, Chung WY, Lee EJ, Jo YS. GLI1 Transcription Factor Affects Tumor Aggressiveness in Patients With Papillary Thyroid Cancers. Medicine (Baltimore). 2015c Jun;94(25):e998. doi: 10.1097/MD.0000000000000998.

Lee JC, Zhao JT, Clifton-Bligh RJ, Gill A, Gundara JS, Ip JC, Glover A, Sywak MS, Delbridge LW, Robinson BG, Sidhu SB. MicroRNA-222 and microRNA-146b are tissue and circulating biomarkers of recurrent papillary thyroid cancer. Cancer. 2013 Dec 15;119(24):4358-65. doi: 10.1002/cncr.28254.

Li B, Dewey CN. RSEM: accurate transcript quantification from RNA-Seq data with or without a reference genome. BMC Bioinformatics. 2011 Aug 4;12:323. doi: 10.1186/1471-2105-12-323.

Peer] reviewing PDF | (2015:10:7164:2:1:NEW 11 May 2016) 
504

505

506

507

508

509

510

511

512

513

514

515

516

517

518

519

520

521

522

523

524

525

526

527

528

529

530

531

532

533

534

535

536

537

538

539

540

541

542

543

544

545

546

547

548

549

550

551

552

Li W, Jin X, Zhang Q, Zhang G, Deng X, Ma L. Decreased expression of miR-204 is associated with poor prognosis in patients with breast cancer. Int J Clin Exp Pathol. 2014 May 15;7(6):3287-92. eCollection 2014.

Liu L, Wang J, Li X, Ma J, Shi C, Zhu H, Xi Q, Zhang J, Zhao X, Gu M. MiR-204-5p suppresses cell proliferation by inhibiting IGFBP5 in papillary thyroid carcinoma. Biochem Biophys Res Commun. 2015 Feb 20;457(4):621-6. doi: 10.1016/j.bbrc.2015.01.037.

Ma L, Huang Y, Zhu W, Zhou S, Zhou J, Zeng F, Liu X, Zhang Y, Yu J. An integrated analysis of miRNA and mRNA expressions in non-small cell lung cancers. PLoS One. 2011;6(10):e26502. doi: 10.1371/journal.pone.0026502.

Mambo E, Chatterjee A, Xing M, Tallini G, Haugen BR, Yeung SC, Sukumar S, Sidransky D. Tumorspecific changes in mtDNA content in human cancer. Int J Cancer. 2005 Oct 10;116(6):920-4.

Maragkakis M, Reczko M, Simossis VA, Alexiou P, Papadopoulos GL, Dalamagas T, Giannopoulos G, Goumas G, Koukis E, Kourtis K, Vergoulis T, Koziris N, Sellis T, Tsanakas P, Hatzigeorgiou AG. DIANA-microT web server: elucidating microRNA functions through target prediction. Nucleic Acids Res. 2009 Jul;37(Web Server issue):W273-6. doi: 10.1093/nar/gkp292.

Nilubol N, Sukchotrat C, Zhang L, He M, Kebebew E. Molecular pathways associated with mortality in papillary thyroid cancer. Surgery. 2011 Dec;150(6):1023-31. doi: 10.1016/j.surg.2011.09.021.

Nunez-Iglesias J, Liu CC, Morgan TE, Finch CE, Zhou XJ. Joint genome-wide profiling of miRNA and mRNA expression in Alzheimer's disease cortex reveals altered miRNA regulation. PLoS One. 2010 Feb 1;5(2):e8898. doi: 10.1371/journal.pone.0008898.

Otto T, Fandrey J. Thyroid hormone induces hypoxia-inducible factor 1alpha gene expression through thyroid hormone receptor beta/retinoid $\mathrm{x}$ receptor alpha-dependent activation of hepatic leukemia factor. Endocrinology. 2008 May;149(5):2241-50. doi: 10.1210/en.2007-1238.

Pallante P, Visone R, Ferracin M, Ferraro A, Berlingieri MT, Troncone G, Chiappetta G, Liu CG, Santoro M, Negrini M, Croce CM, Fusco A. MicroRNA deregulation in human thyroid papillary carcinomas. Endocr Relat Cancer. 2006 Jun;13(2):497-508.

Pizzini S, Bisognin A, Mandruzzato S, Biasiolo M, Facciolli A, Perilli L, Rossi E, Esposito G, Rugge M, Pilati P, Mocellin S, Nitti D, Bortoluzzi S, Zanovello P. Impact of microRNAs on regulatory networks and pathways in human colorectal carcinogenesis and development of metastasis. BMC Genomics. 2013 Aug 29;14:589. doi: 10.1186/1471-2164-14-589.

R Development Core Team (2008). R: A language and environment for statistical computing. R Foundation for Statistical Computing, Vienna, Austria. ISBN 3-900051-07-0, URL http://www.R-project.org.

Rossing M, Borup R, Henao R, Winther O, Vikesaa J, Niazi O, Godballe C, Krogdahl A, Glud M, HjortSørensen C, Kiss K, Bennedbæk FN, Nielsen FC. Down-regulation of microRNAs controlling tumourigenic factors in follicular thyroid carcinoma. J Mol Endocrinol. 2012 Jan 25;48(1):11-23. doi: 10.1530/JME-11-0039.

Rustin P. Mitochondria, from cell death to proliferation. Nat Genet. 2002 Apr; 30(4):352-3.

Schvartz C, Bonnetain F, Dabakuyo S, Gauthier M, Cueff A, Fieffé S, Pochart JM, Cochet I, Crevisy E, Dalac A, Papathanassiou D, Toubeau M. Impact on overall survival of radioactive iodine in low-risk differentiated thyroid cancer patients. J Clin Endocrinol Metab. 2012 May;97(5):1526-35. doi: 10.1210/jc.2011-2512.

Shi W, Gerster K, Alajez NM, Tsang J, Waldron L, Pintilie M, Hui AB, Sykes J, P'ng C, Miller N, McCready D, Fyles A, Liu FF. MicroRNA-301 mediates proliferation and invasion in human breast cancer. Cancer Res. 2011 Apr 15;71(8):2926-37. doi: 10.1158/0008-5472.CAN-10-3369.

Slaby O, Svoboda M, Fabian P, Smerdova T, Knoflickova D, Bednarikova M, Nenutil R, Vyzula R. Altered expression of miR-21, miR-31, miR-143 and miR-145 is related to clinicopathologic features of colorectal cancer. Oncology. 2007;72(5-6):397-402. doi: 10.1159/000113489.

Sobin LH, Wittekind Ch., eds. UICC: TNM classification of malignant tumors, 6th ed. New York: WileyLiss, 2002.

PeerJ reviewing PDF | (2015:10:7164:2:1:NEW 11 May 2016) 
Sun Y, Yu S, Liu Y, Wang F, Liu Y, Xiao H. Expression of miRNAs in Papillary Thyroid Carcinomas Is Associated with BRAF Mutation and Clinicopathological Features in Chinese Patients. Int J Endocrinol. 2013;2013:128735. doi: 10.1155/2013/128735.

Swierniak M, Wojcicka A, Czetwertynska M, Stachlewska E, Maciag M, Wiechno W, Gornicka B, Bogdanska M, Koperski L, de la Chapelle A, Jazdzewski K. In-depth characterization of the microRNA transcriptome in normal thyroid and papillary thyroid carcinoma. J Clin Endocrinol Metab. 2013 Aug;98(8):E1401-9. doi: 10.1210/jc.2013-1214.

Tetzlaff MT, Liu A, Xu X, Master SR, Baldwin DA, Tobias JW, Livolsi VA, Baloch ZW. Differential expression of miRNAs in papillary thyroid carcinoma compared to multinodular goiter using formalin fixed paraffin embedded tissues. Endocr Pathol. 2007 Fall;18(3):163-73.

Wang J, Lu M, Qiu C, Cui Q. TransmiR: a transcription factor-microRNA regulation database. Nucleic Acids Res. 2010a;38:D119-D122. doi: 10.1093/nar/gkp803.

Wang YX, Zhang XY, Zhang BF, Yang CQ, Chen XM, Gao HJ. Initial study of microRNA expression profiles of colonic cancer without lymph node metastasis. J Dig Dis. 2010b Feb;11(1):50-4. doi: 10.1111/j.1751-2980.2009.00413.x.

Wojtas B, Ferraz C, Stokowy T, Hauptmann S, Lange D, Dralle H, Musholt T, Jarzab B, Paschke R, Eszlinger M. Differential miRNA expression defines migration and reduced apoptosis in follicular thyroid carcinomas. Mol Cell Endocrinol. 2014 May 5;388(1-2):1-9. doi: 10.1016/j.mce.2014.02.011.

Yan LX, Huang XF, Shao Q, Huang MY, Deng L, Wu QL, Zeng YX, Shao JY. MicroRNA miR-21 overexpression in human breast cancer is associated with advanced clinical stage, lymph node metastasis and patient poor prognosis. RNA. 2008 Nov;14(11):2348-60. doi: 10.1261/rna.1034808.

Yang Z, Yuan Z, Fan Y, Deng X, Zheng Q. Integrated analyses of microRNA and mRNA expression profiles in aggressive papillary thyroid carcinoma. Mol Med Rep. 2013 Nov;8(5):1353-8. doi: $10.3892 / \mathrm{mmr} .2013 .1699$.

Yin Y, Zhang B, Wang W, Fei B, Quan C, Zhang J, Song M, Bian Z, Wang Q, Ni S, Hu Y, Mao Y, Zhou L, Wang Y, Yu J, Du X, Hua D, Huang Z. miR-204-5p inhibits proliferation and invasion and enhances chemotherapeutic sensitivity of colorectal cancer cells by downregulating RAB22A. Clin Cancer Res. 2014 Dec 1;20(23):6187-99. doi: 10.1158/1078-0432.CCR-14-1030.

Yip L, Kelly L, Shuai Y, Armstrong MJ, Nikiforov YE, Carty SE, Nikiforova MN. MicroRNA signature distinguishes the degree of aggressiveness of papillary thyroid carcinoma. Ann Surg Oncol. 2011 Jul;18(7):2035-41. doi: 10.1245/s10434-011-1733-0. 


\section{Table 1 (on next page)}

Patient Characteristics and Integrated Profiles in the TCGA PTC Cohort 
1 Table 1. Patient Characteristics and Integrated Profiles in the TCGA PTC Cohort

\begin{tabular}{|c|c|c|c|c|}
\hline \multirow[t]{2}{*}{ Variables } & \multirow{2}{*}{$\begin{array}{l}\text { PTC LNN } \\
\text { N0 } \\
(n=213)\end{array}$} & \multicolumn{3}{|l|}{ PTC LNM-P } \\
\hline & & $\begin{array}{l}\text { N1 } \\
(\mathrm{n}=53)\end{array}$ & $\begin{array}{l}\text { N1a } \\
(n=86)\end{array}$ & $\begin{array}{l}\text { N1b } \\
(n=66)\end{array}$ \\
\hline Age range (years) & $15-85$ & $19-83$ & $18-83$ & $19-89$ \\
\hline Mean age & 49.4 & 41.9 & 43.5 & 48.4 \\
\hline \multicolumn{5}{|l|}{ Gender (n) } \\
\hline Male & $50(23.5 \%)$ & $14(26.4 \%)$ & $25(29.1 \%)$ & $27(40.9 \%)$ \\
\hline Female & $163(76.5 \%)$ & $39(73.6 \%)$ & $61(70.9 \%)$ & $39(59.1 \%)$ \\
\hline \multicolumn{5}{|l|}{ Disease free status } \\
\hline Recurred/progressed & $5(2.3 \%)$ & $7(13.2 \%)$ & $6(7 \%)$ & $6(9.1 \%)$ \\
\hline Disease free & $178(83.6 \%)$ & $41(77.4 \%)$ & $75(87.2 \%)$ & $47(71.2 \%)$ \\
\hline Unknown & $30(14.1 \%)$ & $5(9.4 \%)$ & $5(5.8 \%)$ & $13(19.7 \%)$ \\
\hline Disease free (range in months) & $0.03-155$ & $0-131$ & $0-157$ & $0.2-46$ \\
\hline Mean disease free survival & $\begin{array}{l}23.6 \\
(n=183)\end{array}$ & $\begin{array}{l}34.5 \\
(n=48)\end{array}$ & $\begin{array}{l}21.5 \\
(\mathrm{n}=81)\end{array}$ & $\begin{array}{l}13.5 \\
(\mathrm{n}=53)\end{array}$ \\
\hline \multicolumn{5}{|l|}{ Overall survival status } \\
\hline Deceased & $35(16.4 \%)$ & $12(22.6 \%)$ & $11(12.8 \%)$ & $19(28.8 \%)$ \\
\hline Alive & $178(83.6 \%)$ & $41(77.6 \%)$ & $75(87.2 \%)$ & $47(71.2 \%)$ \\
\hline Overall survival (range in months) & $0.03-155$ & $0-131$ & $0-157$ & $0.2-97.7$ \\
\hline Mean overall survival & $\begin{array}{l}24.3 \\
(\mathrm{n}=182)\end{array}$ & $\begin{array}{l}35.2 \\
(n=43)\end{array}$ & $\begin{array}{l}21.4 \\
(\mathrm{n}=75)\end{array}$ & $\begin{array}{l}15.2 \\
(\mathrm{n}=50)\end{array}$ \\
\hline \multicolumn{5}{|l|}{ Extrathyroidal extension } \\
\hline None & $160(75.1 \%)$ & $31(58.5 \%)$ & $49(57 \%)$ & $37(56.1 \%)$ \\
\hline Minimal (T3) & $42(19.7 \%)$ & $14(26.4 \%)$ & $33(38.4 \%)$ & $23(34.8 \%)$ \\
\hline Moderate/ advanced (T4a) & $3(1.4 \%)$ & $5(9.4 \%)$ & $1(1.2 \%)$ & $4(6.1 \%)$ \\
\hline Very advanced (T4b) & $0(0 \%)$ & $1(1.9 \%)$ & $0(0 \%)$ & $0(0 \%)$ \\
\hline Unknown & $8(3.8 \%)$ & $2(3.8 \%)$ & $3(3.5 \%)$ & $2(3 \%)$ \\
\hline \multicolumn{5}{|l|}{ BRAF status } \\
\hline Mutated & $94(44.1 \%)$ & $25(47.2 \%)$ & $53(61.6 \%)$ & $32(48.9 \%)$ \\
\hline Wild type & $119(55.9 \%)$ & $28(52.8 \%)$ & $33(38.4 \%)$ & $34(51.5 \%)$ \\
\hline
\end{tabular}

2 


\section{1}

\section{Survival analysis of PTC with LNM and PTC without LNM}

A

Overall survival

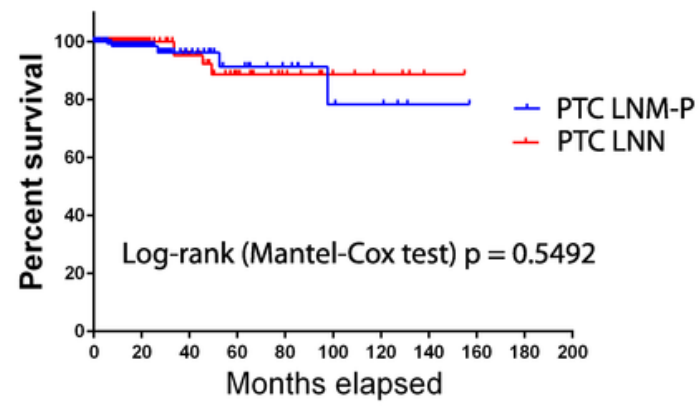

\section{B Disease-free survival}

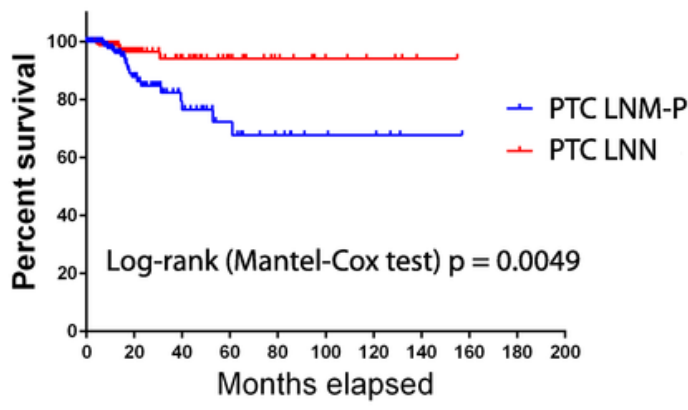


2

Heat Map of the 181 Differentially Expressed miRNAs in PTC LNM-P and LNN (Student's T-test with $\mathrm{BH}$ corrected $\mathrm{p}$ value $\leq 0.05$ ). 


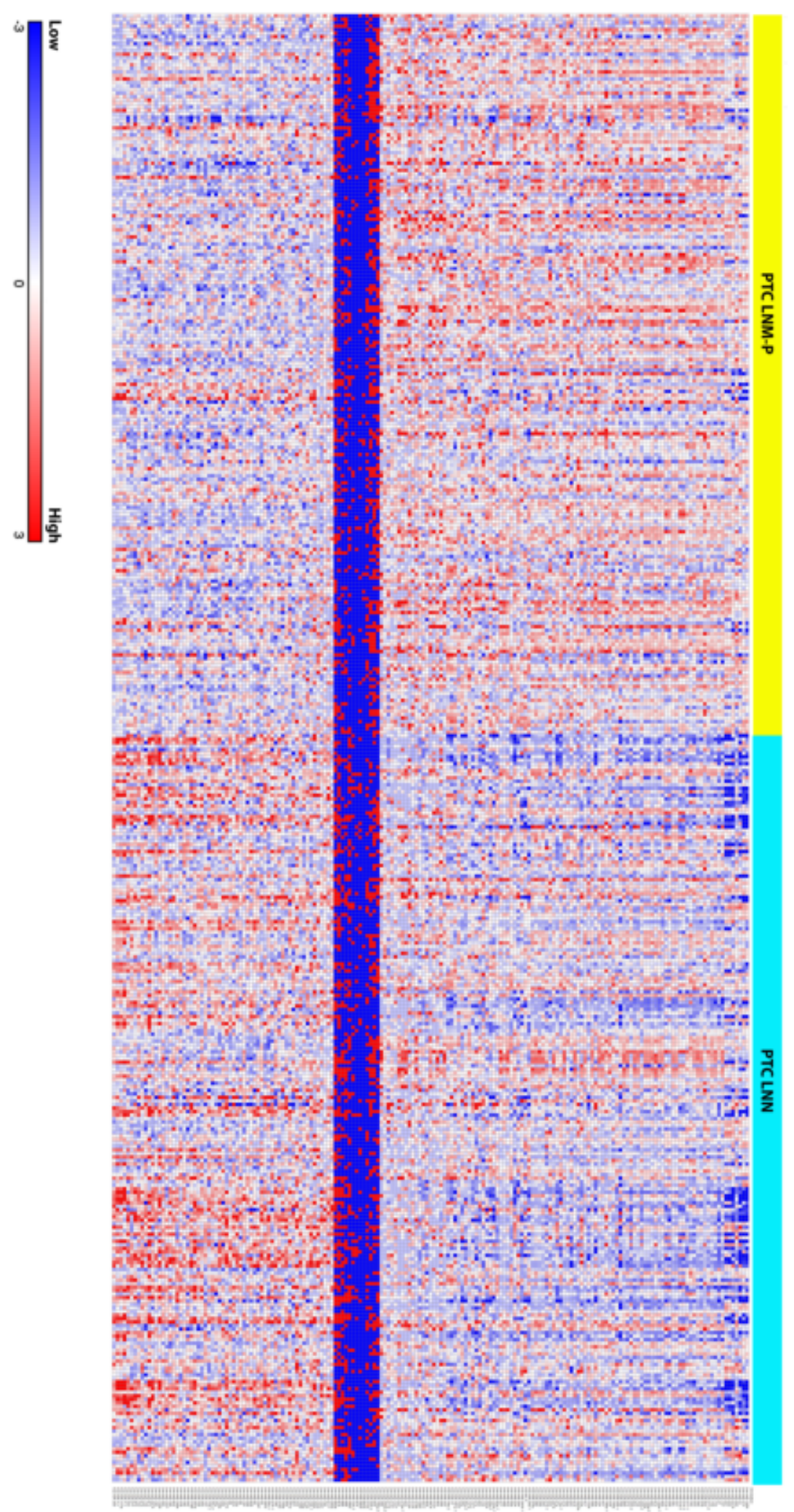




\section{3}

Expression levels of five selected miRNAs deregulated in PTC.

Boxplots (A) illustrate log2 normalized miRNA reads in PTC LNM-P, PTC LNN and normal thyroid. Table (B) showing log2 fold change and $p$ value of selected miRNAs in PTC LNM-P compared to PTC LNN.

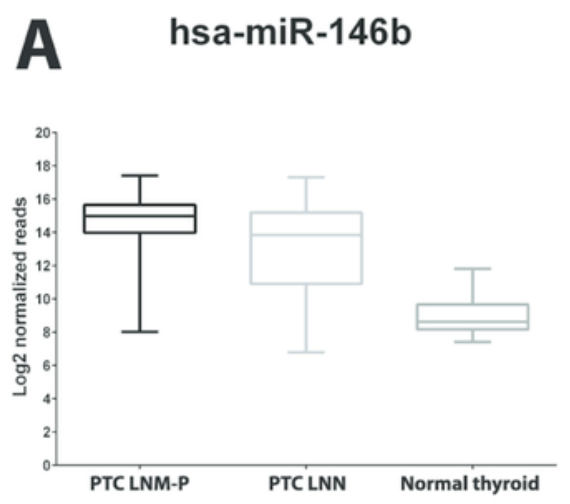

hsa-miR-375

hsa-miR-31

hsa-miR-204
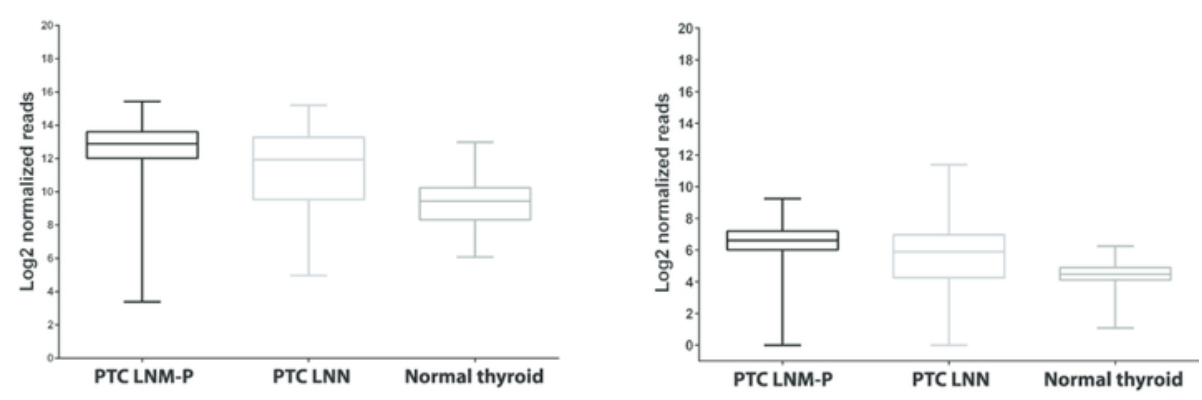

hsa-miR-7-2
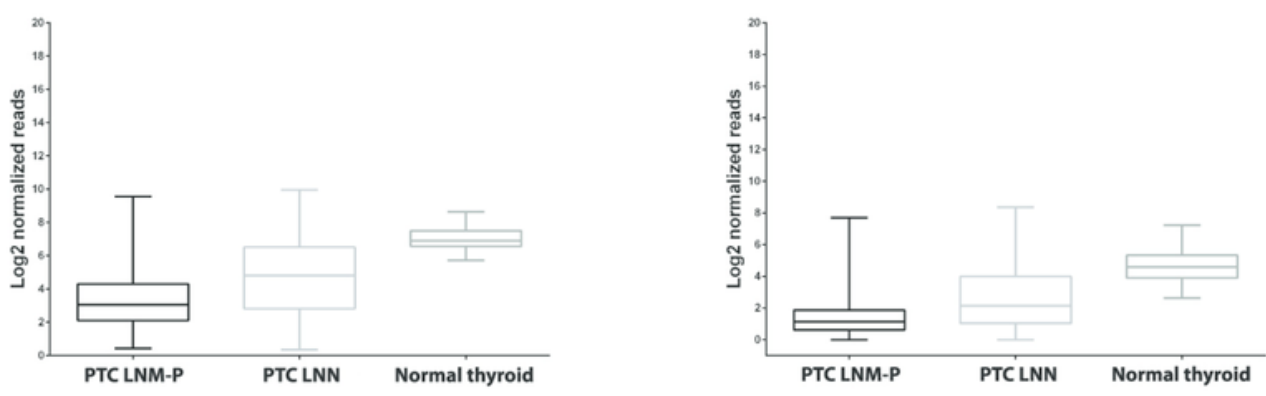

B

\begin{tabular}{lll}
\hline miRNA ID & $\mathbf{L o g}_{2}$ fold change & BH adj. $\mathbf{p}$ value \\
\hline hsa-miR-146b & 1.7 & $1.41 \mathrm{E}-03$ \\
hsa-miR-375 & 1.3 & $3.00 \mathrm{E}-02$ \\
hsa-miR-31 & 1.0 & $4.59 \mathrm{E}-09$ \\
hsa-miR-7-2 & -1.1 & $3.00 \mathrm{E}-02$ \\
hsa-miR-204 & -1.3 & $2.11 \mathrm{E}-05$ \\
\hline Student's T-test with BH corrected p-value $\leq 0.05 ; \log _{2}$ fold change $\geq 1$ or $\leq-1$
\end{tabular}


4

Significantly enriched pathways in PTCs.

Significant KEGG pathway associations to 8611 significantly deregulated genes in PTC LNM-P versus PTC LNN, 14192 genes in PTC LNM-P versus normal thyroid and 13392 genes in PTC LNN versus normal thyroid.

A PtC LnM-P vs PTC LnN Oxidative phosphorylation
Cell adhesion molecules (CAMS) Cell achesion molecules (CAMs) Cytokine-cytokine receptor interaction

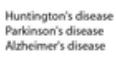

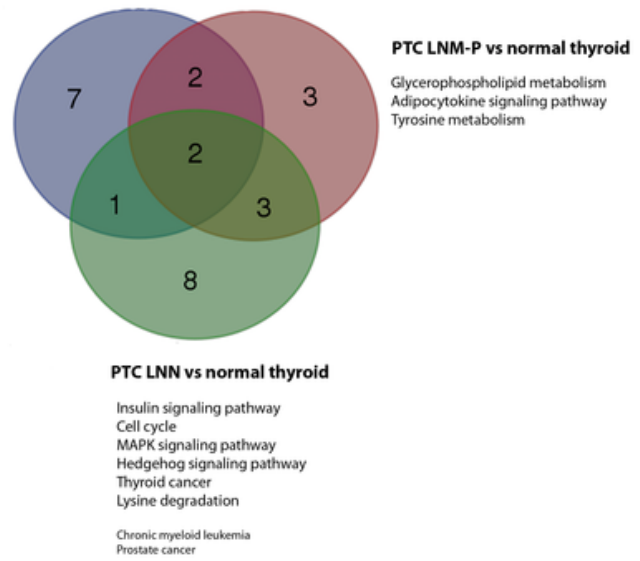

B

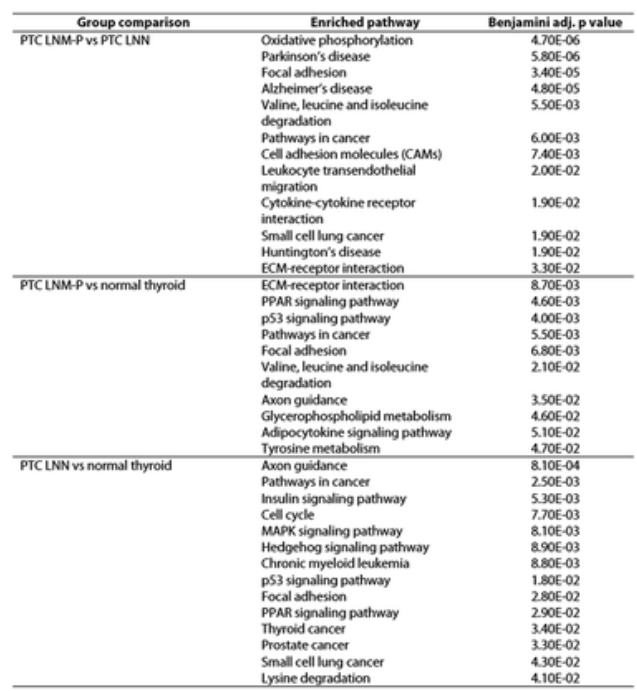


5

KEGG pathway map illustrating oxidative phosphorylation in human.

The OxPhos-related genes significantly altered in PTC LNM-P compared to PTC LNN were depicted with red star. Pathway figure was obtained from KEGG (kyoto encyclopedia of genes and genomes) (Kanehisa and Goto, 2000; Kanehisa et al., 2016) in DAVID analysis.

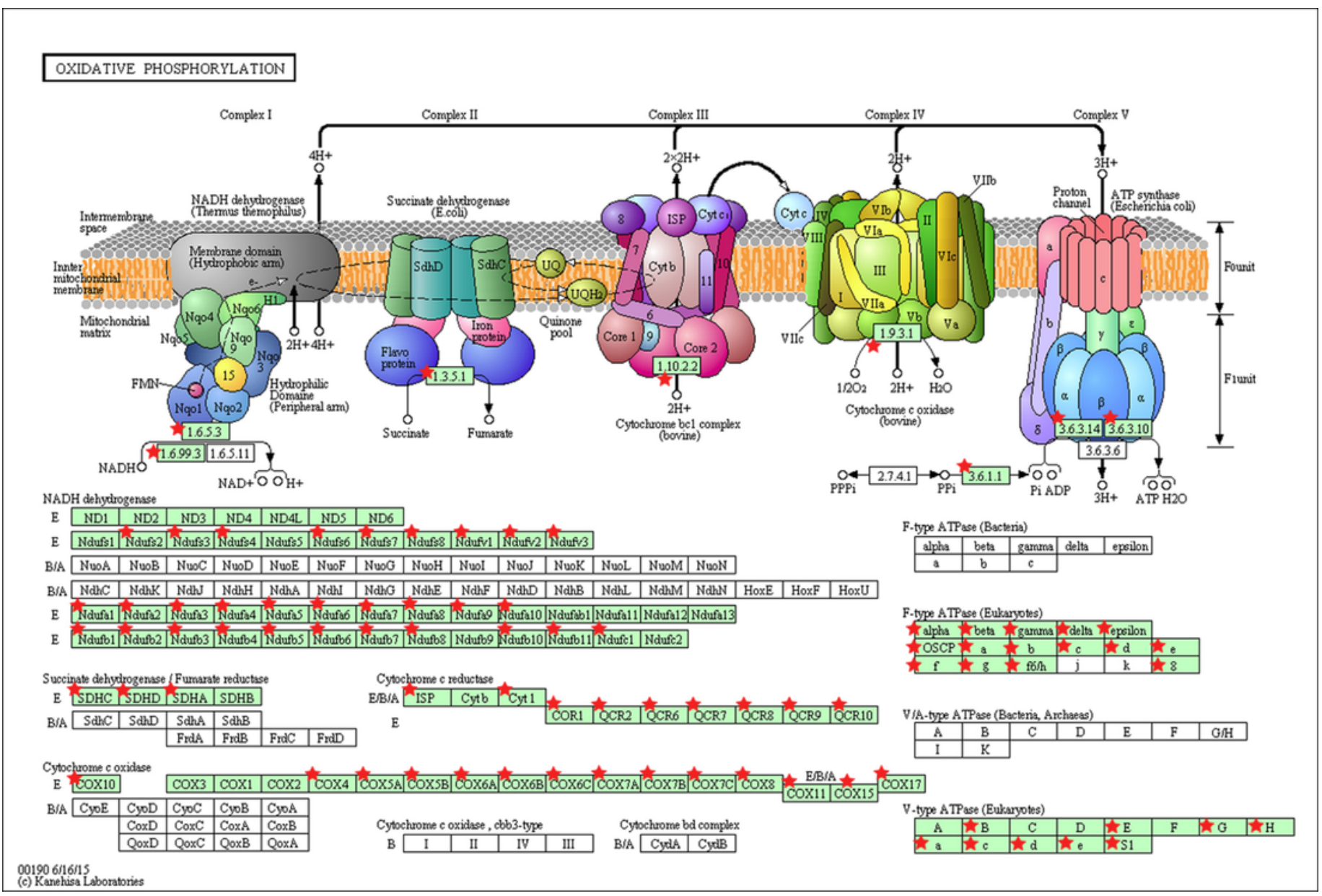




\section{6}

Grand view of top 200 regulatory circuits constructed using significantly dysregulated miRNAs and genes in PTC LNM-P compared to PTC LNN.

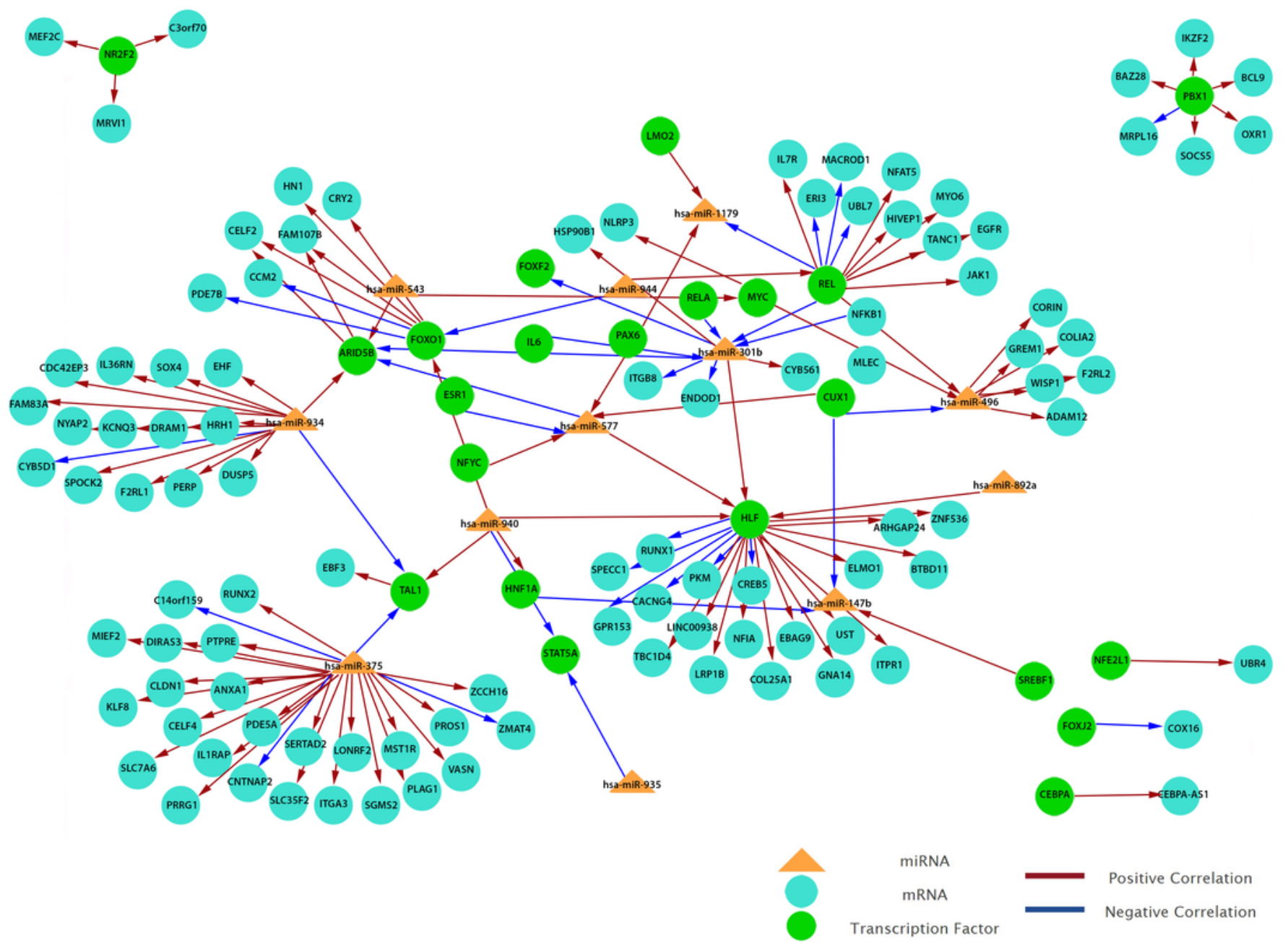

